\title{
Scalability Aware Energy Consumption and Dissipation Models for Wireless Sensor Networks
}

\author{
Mohamed El Fissaoui ${ }^{1}$, Said Benkirane ${ }^{2}$, Abderrahim Beni-Hssane ${ }^{3}$, Mostafa Saadi ${ }^{4}$ \\ ${ }^{1,3}$ LAROSERI Laboratory, Computer Science Department, Sciences Faculty, Chouaïb Doukkali University El Jadida, \\ Morocco \\ ${ }^{2}$ High School of Technology, Essaouira, Cadi Ayyad University, Morocco \\ ${ }^{4}$ Département Informatique \& Télécoms Ecole Nationale des Sciences Appliquées(ENSA), \\ Khouribga Université Hassan 1er - Settat, Morocco
}

\section{Article Info}

Article history:

Received Jul 29, 2016

Revised Nov 20, 2016

Accepted Dec 5, 2016

\section{Keyword:}

Energy consumption

Energy dissipation models

Wireless sensor networks

\begin{abstract}
Most of Wireless Sensor Networks researches focus on reducing the amount of energy consumed by nodes and network to increase the network lifetime. Thus, several papers have been presented and published to optimize energy consumption in each area of WSNs, such as routing, localization, coverage, security, etc. To test and evaluate their propositions, authors apply an energy dissipation model; this model must be more realistic and suitable to give good results. In this paper we present a general preview on different sources of energy consumption in wireless sensor networks, and provide a comparative study between two energy models used in WSNs that offer an effective and an adequate tool for researchers.
\end{abstract}

Copyright @ 2017 Institute of Advanced Engineering and Science. All rights reserved.

Corresponding Author:

Mohamed El Fissaoui,

LAROSERI laboratory,

Computer Science Department,

Faculty of Sciences,

Chouaïb Doukkali University,

Route Ben Maachou, 24000, El Jadida, Morocco.

Email: Elfissaoui.m@ucd.ac.ma

\section{INTRODUCTION}

Wireless sensor networks are formed by a large number of sensor nodes, which are commonly known as motes forming wireless ad-hoc networks. These motes are small in size and have limited processing power, memory and battery life. Motes typically have sensors such as thermometers attached to them in order to gather data about the physical environment [1].

A critical constraint in sensor networks is that sensor nodes employ batteries. The second constraint is that sensors will be deployed unattended and in large numbers, so it's difficult to change or recharge sensors' batteries. Therefore, all systems, processes and communication protocols for sensor networks must minimize the power consumption. Some of the most challenging issues that have been studied in different areas of WSNs are the medium access [2], routing strategies, clustering schemes [3], and application layer dynamics, etc. All these approaches contribute to reduce the energy consumption and increase the network lifetime [4].

Simulation plays a very important role, it permits to test and to evaluate the proposed solutions by researchers in different domains, thus it should provides correct measurements as those that will be gotten in real-world environment. Therefore, the energy model used must be more realistic and more suitable to give accurate results. 
The rest of this paper is organized as follows. Section II presents different sources of energy consumption in WSNs. Section III exhibits some energy consumption models used. Section IV evaluates the performance of two energy models by simulations. Finally, Section V gives concluding remarks.

\section{SOURCES OF ENERGY CONSUMPTION}

\subsection{Normal Sources}

In WSNs energy consumption occurs in three domains: sensing, data processing, and communications [5]. Sensor node expends maximum energy in data communication (Emission and Reception) than data processing or sensing (Figure 1). Therefore, to save energy and prolong network lifetime, we should consider how to minimize communication costs. In addition, any method which considers these problems needs to maintain network stability [6], [7].

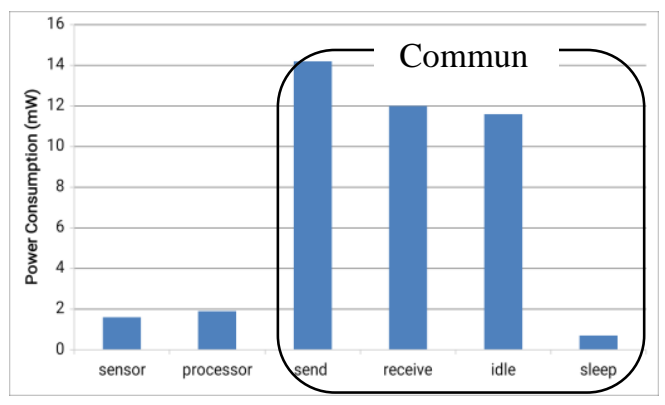

Figure 1. Energy Consumption in WSNs

\subsection{Additional Sources}

In addition to sources mentioned above, there are some other unexpected sources that are considered as sources of energy wasting. The major sources of energy waste are:

a) Collisions: Occurs when two or more nodes attempt to transmit a packet across the network at the same time. The transmitted packets must be discarded and then retransmitted, thus the retransmission of those packets increases the energy consumption and the latency.

b) Idle listening: Listening to receive possible traffic that is not sent (major source of energy consumption). Usually a node in a WSN doesn't know when to wake up to receive a packet, thus it must keep its radio ON which consumes most of the energy.

c) Overhearing: When nodes receive a packet for another node. Overhearing can be a major reason of energy waste mainly with a high node density causing a heavy traffic load.

d) Protocol overhead: Control frames do not carry useful information although their transmission consumes energy.

e) Adaptation - Reconfiguring: When nodes join and leave the network it needs to be known in the network (Neighboring, Routing, localization...)

f) Overmitting: means that a transmission takes place while the intended receiver is not ready to receive. Overmitting is common in asynchronous protocols using preamble sampling.

g) Computational for location: If nodes are equipped with localization device (GPS) it consumes more energy, if not, it uses some techniques to be localized and thus it consumes also the energy.

h) Control Packet Overhead: Sending and receiving control packets consumes energy too, and less useful data packets can be transmitted.

\section{ENERGY CONSUMPTION MODELS}

\subsection{Network Model}

We consider a wireless sensor network with $\mathrm{N}$ nodes randomly distributed in a field (Figure 2). We assume that nodes always have data to transmit to the base station, which is often far from the sensing area. The network is organized into a clustering hierarchy, and the cluster-heads execute fusion function to reduce correlated data produced by the sensor nodes within the clusters. The cluster-heads $(\mathrm{CH})$ transmit the aggregated data to the base station directly. We assume that all nodes are homogeneous, stationary and equipped with initial energy of $E_{0}$. 


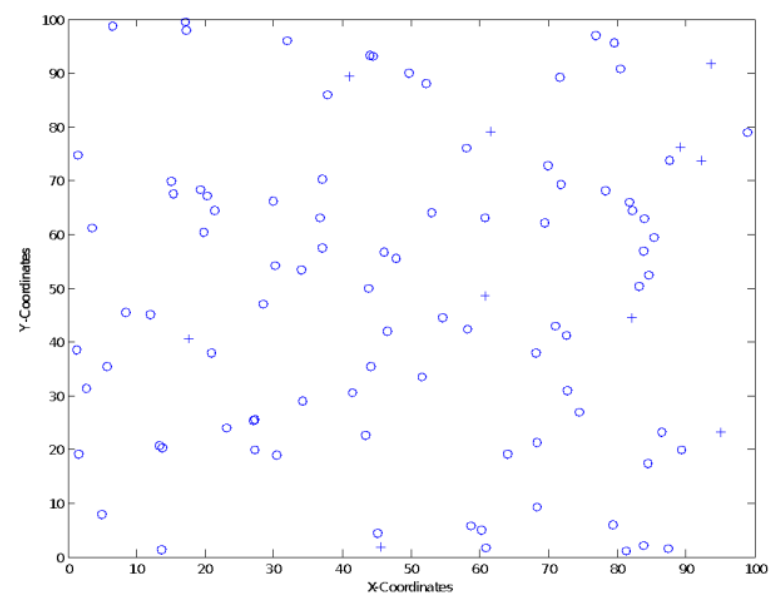

Figure 2. The network with 100 nodes randomly deployed (o Normal node, + Cluster head)

\subsection{Energy Models}

a) Model proposed by Heinzelman et al.

According to the radio energy dissipation model proposed by Heinzelman et al. [8] the energy expended by the radio is given by:

$$
\operatorname{ETx}(L, d)=\left\{\begin{array}{cc}
L E_{\text {elec }}+L_{\epsilon f s} d^{2}, & \mathrm{~d}<d_{0} \\
L E_{\text {elec }}+L_{\epsilon m p} d^{4}, & d \geq d_{0}
\end{array}\right.
$$

Where $\mathrm{L}$ is the number of bits in every packet, $E_{\text {elec }}$ is the energy dissipated per bit to run the transmitter $E_{T x}$ or the receiver $E_{R x}$ circuit, $\epsilon_{f s}$ is the free space fading energy, $\epsilon_{m p}$ is the multi-path fading energy and $\mathrm{d}$ is the distance between the sender and the receiver and $d_{0}$ distance fixed by the model (Figure 3).

To receive this message the radio expends energy:

$$
E_{R x}(L)=L E_{\text {elec }}
$$

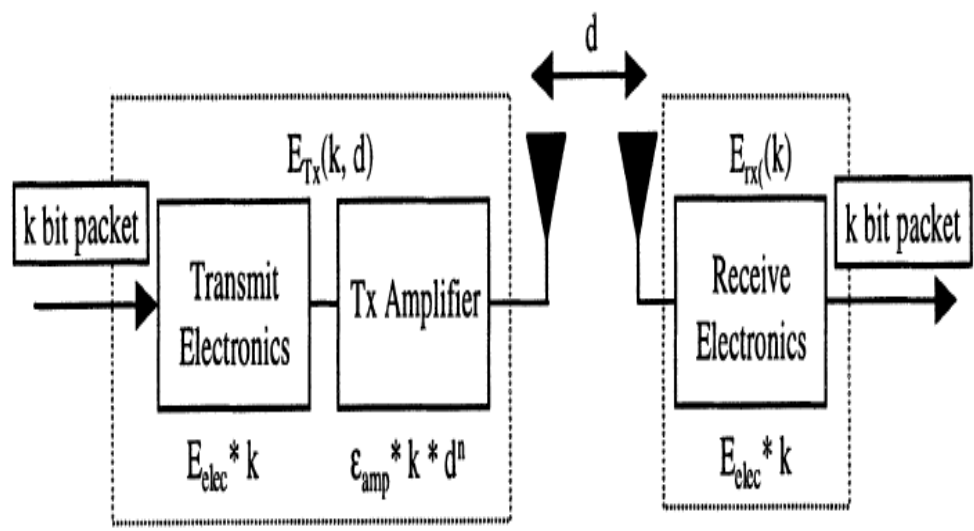

Figure 3. Radio Energy Dissipation Model

Total energy during each round is given by:

$$
E_{\text {Round }}=L\left[2 N E_{\text {elec }}+N E_{D A}+k_{\epsilon m p} d_{t o B S}^{4}+N_{\epsilon f S} d_{\text {toCH }}^{2}\right]
$$


Where $\mathrm{k}$ is the number of clusters, $E_{D A}$ is the data aggregation cost expended in $C H, d_{t o B S}$ is the average distance between the cluster-head and the base station and $d_{t o C H}$ is the average distance between cluster members and the cluster-head.

The optimal number of clusters is:

$$
K_{o p t}=\frac{\sqrt{E_{f s}}}{\sqrt{E_{m p}}} \frac{\sqrt{N}}{\sqrt{2 \pi}} \frac{M}{d_{\text {toBS }}^{2}}
$$

The model proposed considers microcontroller processing and radio transmission and receiving only. This model does not consider other important sources of energy consumption, such as transient energy, sensor sensing, sensor logging and actuation.

b) Model proposed by Shih et al.

Shih et al. [9] presented a model developed for $\mu$ AMPS a specific wireless sensor node. The authors present a formulation for the radio power consumption $(\mathrm{Pc})$ as:

$$
P_{c}=N_{T}\left[P_{T}\left(T_{\text {on }}+T_{s t}\right)+P_{\text {out }} T_{\text {on }}\right]+N_{R}\left[P_{R}\left(R_{\text {on }}+R_{\text {st }}\right)\right]
$$

Where PT is the power consumed by the transmitter/receiver, Pout is the output power of the transmitter, Ron is the transmitter/receiver on time, Rst is the transmitter/receiver startup time and NT is the number of times transmitter/receiver is switched on per unit time, which depends on the task and Medium Access Control (MAC) scheme used. Ton can further be rewritten as L/R, where L is the packet size and R is the data rate.

The $\mu$ AMPS model doesn't specify the power consumed in transmitting or receiving one bit.

Hill et al. propose in [10] how calculate the energy required for transmitting one bit.

Time to send or receive one bit $=1 / 1 \mathrm{Mbps}=1 \mu \mathrm{sec}$ :

$$
\text { Energy }=\text { Power } \times \text { Time }
$$

Where Power is in Watts and Time is in seconds

c) Mica2 Specific Model

Polastre et al. [11] proposed a model that presents the total energy consumption for Mica2 as the summation of energy transmitting, receiving, listening, sampling data and sleeping.

As the authors present current consumption and time, and assuming that Mica2 is powered by a $3 \mathrm{~V}$ source [4], one can calculate energy in transmitting and receiving one bit, as:

$$
\text { Energy }=\text { Current } \times \text { Voltage } \times \text { Time }
$$

where current is in Amperes, Voltage is in Volts and Time is in seconds.

$$
\begin{aligned}
& \text { Energy }_{T x}=20 \times 10-3 \mathrm{~A} \times 3 \text { Volts } \times 416 \times 10-6 \mathrm{sec} / 8 \text { bits } \\
& \text { Thus: Energy }{ }_{T x}=3.12 \mu \mathrm{J} / \mathrm{bit} \\
& \text { Energy }_{R x}=15 \times 10-3 \mathrm{~A} \times 3 \text { Volts } \times 416 \times 10-6 \mathrm{sec} / 8 \text { bits } \\
& \text { Thus: Energy }{ }_{R x}=2.34 \mu \mathrm{J} / \mathrm{bit}
\end{aligned}
$$

This model does not consider other important sources of energy consumption, such as transient energy, sensor logging and actuation.

d) Mica2 Specific Model with actual measurements platform.

Shnayder et al. [12] presented a current consumption model based on measurements on the Mica2

Energy cost per bit transmitted is:

Energy $_{T x}=(8+21.5) \times 10-3 \mathrm{~A} \times 3$ Volts $\times 416 \times 10-6 \mathrm{sec} / 8$ bits

Thus: Energy ${ }_{T x}=4.602 \mu \mathrm{J} / \mathrm{bit}$

Energy $_{R x}=(8+7) \times 10-3 \mathrm{~A} \times 3$ Volts $\times 416 \times 10-6 \mathrm{sec} / 8 \mathrm{bits}$

Thus: Energy ${ }_{R x}=2.34 \mu \mathrm{J} / \mathrm{bit}$ 
e) Model proposed by Miller et al.

Miller et al [13] propose a model based on the Mica2 Motes, this energy model take in consideration the time and power required for the radio to transition from the sleep state to idle and from the idle state to sleep. In practice, these values are non negligible, but not accounted for in most previous work.

Considered values are:

$$
\begin{aligned}
& T_{\text {trans-on }}=2450 \mu \mathrm{s} \\
& T_{\text {trans-off }}=250 \mu \mathrm{s} \\
& P_{\text {trans-on }}=30 \mathrm{~mW} \\
& P_{\text {trans-off }}=30 \mathrm{~mW}
\end{aligned}
$$
actuation.

The model proposed does not consider energy consumption of sensor sensing, sensor logging and

f) Halgamuge et al.

Halgamuge et al [14] propose a model that takes in consideration other sources of energy consumption such as transient energy $\left(E_{\text {trans }}\right)$, sensor sensing $\left(E_{\text {sens }}\right)$, sensor logging $\left(E_{\text {logg }}\right)$ and actuation.

Total energy consumed by a sensor node during each round is:

$$
E_{\text {Node }}=\left[L E_{\text {sensN }}+L E_{\text {logg } N}+L E_{\text {elec }}+L \epsilon_{f s} d_{\text {toCH }}^{2}+E_{\text {transN }}\right]
$$

Total energy consumed by a $\mathrm{CH}$ during each round is:

$$
\begin{aligned}
E_{C H}= & {\left[L E_{\text {sens } C H}+L E_{\text {loggCH}}+L_{1} E_{D A}\left(\frac{N}{K}\right)+h_{2} E_{\text {elec }}\left[L_{1}\left(\frac{N}{K}-1\right)+\left(\gamma L_{2}\right)+L_{2}(1+\gamma)\right]+\right.} \\
& \left.L_{2}(1+\gamma) \epsilon_{m p} d^{4}{ }_{\text {toBS }}+E_{\text {trans } C H}\right]
\end{aligned}
$$

Where:

a. $L_{1}=\mathrm{L} \times$ number of sensors from its own cluster

b. $\gamma \in 0,1,2 \ldots \mathrm{k}-1$

c. $L_{2}$ Number of bits transmitted by $\mathrm{CH}$ to base station (BS).

d. $h_{2}$ Is a weighting factor that applies to a $\mathrm{CH}$ to indicate by how much it consumes more energy than a regular sensor node for energy transmission.

We assume that the energy dissipation for actuation is not performed.

The energy dissipation in a single cluster during each round is given by:

$$
E_{\text {Cluster }}=E_{C H}+\left(\frac{N}{K}-1\right) E_{\text {Node }}
$$

The total energy for k clusters, during each round:

$$
E_{\text {Total }}=k E_{\text {Cluster }}
$$

The optimal number of clusters in this model is given by:

$$
K_{o p t}=\frac{\sqrt{E_{f s}}}{\sqrt{E_{\alpha}}} \frac{\sqrt{N}}{\sqrt{6}} \frac{M}{d_{\text {toBS }}^{2}}
$$

Where:

$$
E_{\alpha}=\left(\epsilon_{m p}+E_{\text {sens } C H}+E_{\operatorname{logg} C H}+E_{\text {trans } C H}\right)
$$

The model proposed here is more realistic and comprehensive energy model for wireless sensor networks.

g) Other models

In [15] authores an energy model for WSNs considering the physical layer and MAC layer parameters by determining the energy consumed per payload bit transferred without error over AWGN channel. 


\section{SIMULATION RESULTS}

We evaluate the performance of two energy models. The first proposed by Heinzelman et al. and the second proposed by Halgamuge et al. using MATLAB software. We consider a wireless sensor network with $\mathrm{N}=100$ nodes randomly distributed in a $100 \mathrm{~m} \times 100 \mathrm{~m}$ field. We assume the base station is far from the sensing region. The radio parameters used in our simulations are shown in Table 1.

Table 1. Radio characteristics used in our simulations

\begin{tabular}{cc}
\hline Parameter & Value \\
\hline$E_{\text {elec }}$ & $50 \mathrm{~nJ} / \mathrm{bit}$ \\
$\epsilon_{f s}$ & $10 \mathrm{pJ} / \mathrm{bit} / \mathrm{m}^{2}$ \\
$\epsilon_{m p}$ & $0.0013 \mathrm{pJ} / \mathrm{bit} / \mathrm{m}^{4}$ \\
$d_{0}$ & $70 \mathrm{~m}$ \\
Message size (L) & $2000 \mathrm{bits}$ \\
$\mathrm{k}$ & $10 \mathrm{Clusters}$ \\
\hline
\end{tabular}

In our simulation we use a hierarchical routing protocol which used cluster based approach in wireless sensor network called LEACH [8].

Using Mica2 Motes hardware values [16] and formulas cited in [14] and Parameter values used in [17] we obtain the following results:

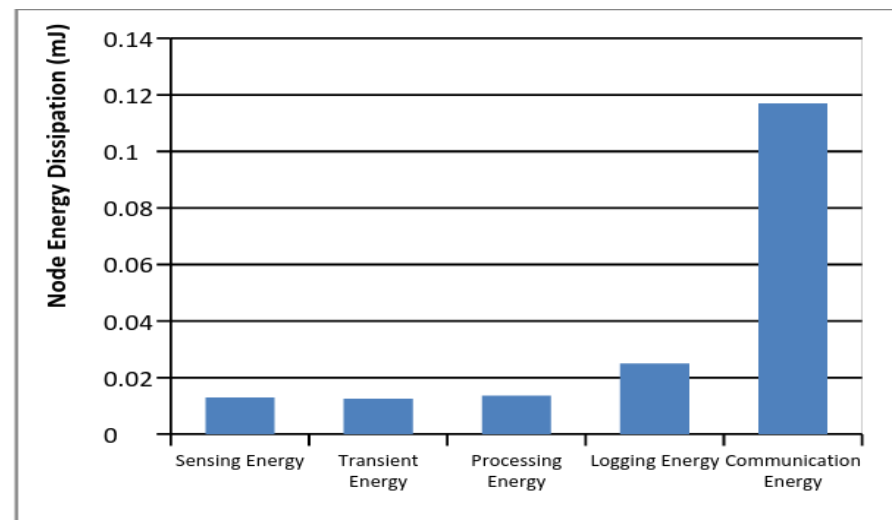

Figure 4. Average Energy Dissipation for every Node

Figure 4 shows the average energy dissipation for every node for all components considered in the model proposed by Halgamuge et al. These energy components are considerable. Therefore they can change results of simulations if they are taken in consideration.

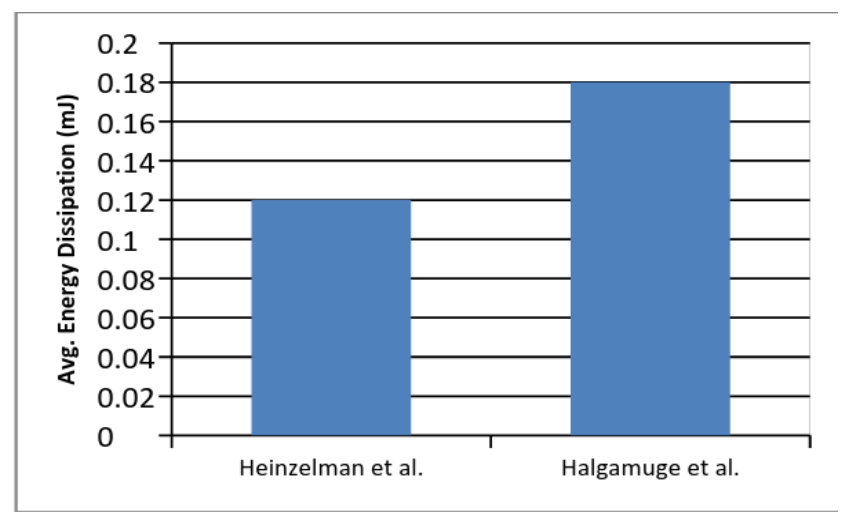

Figure 5. Average Energy Dissipation in two Models 
According to figure 5 above, the average energy dissipation in the model proposed by Halgamuge et al. is heigher as compared with the one proposed by Heinzelman et al. The main reason is that Halgamuge et al. model takes in consideration other sources of energy consumption that are not included in Heinzelman et al. model, i.e., transmit energy, sensor sensing, sensor logging and actuation.

The optimal number of clusters has significant effect on sensor network lifetime. Figure 6 shows that the optimal number of clusters increases with the number of sensors used for two compared energy models. The change is modest in the model proposed by Halgamuge et al. it is nearly stable.

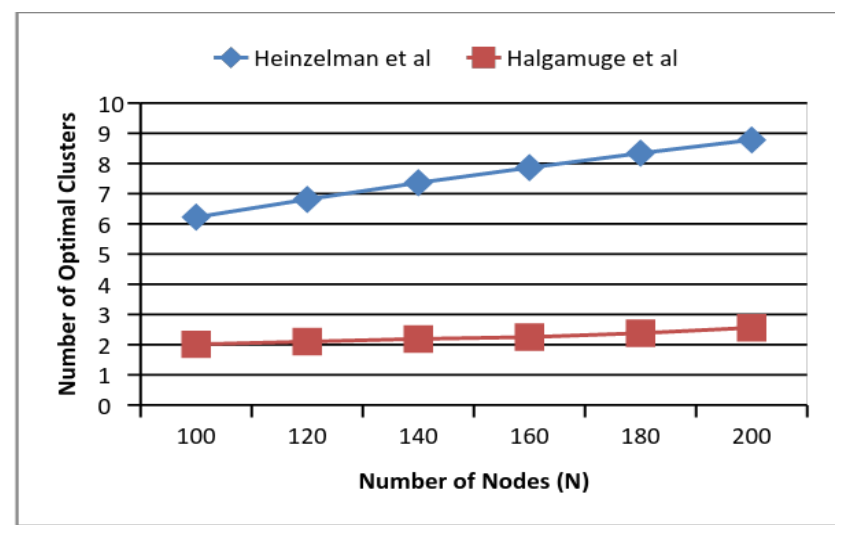

Figure 6. Optimal Number of Clusters Versus Number of Nodes

According to formulas (4) and (15) it is clear that the optimal number of clusters vary with several factors such as distance from $\mathrm{CH}$ to sink node, free space fading energy $\epsilon_{f s}$, square of the sensing field, etc. With each of this factors the optimal number of clusters it is more sensible in Heinzelman et al. model than Halgamuge et al model. That changes significantly simulation results.

Finally Table 2 shows energy sources used in two models compared in this study.

Table 2. Energy Source Considered by two Models

\begin{tabular}{ccc}
\hline Energy Sources & Heinzelman et al. & Halgamuge et al. \\
\hline Processing & $\mathrm{X}$ & $\mathrm{X}$ \\
Communication & $\mathrm{X}$ & $\mathrm{X}$ \\
Sensing & & $\mathrm{X}$ \\
Transeint & & $\mathrm{X}$ \\
Logging & & $\mathrm{X}$ \\
Actuation & & $\mathrm{X}$ \\
Clusters formation & $\mathrm{X}$ & $\mathrm{X}$ \\
\hline
\end{tabular}

\section{CONCLUSION}

In the first part in this paper, we have presented different sources of the energy consumption in wireless sensor networks and major sources of energy waste. In the second part, we have provided several energy models used in WSN, finally, a comparative study is conducted for two energy models proposed by Heinzelman et al. and proposed by Halgamuge et al. It permits to help researchers to choice a suitable and appropriate model to use it in their simulations in order to have good results for their propositions.

In the future work we will provide a large comparative study while take in account other models such as the model proposed by Zhu et al. [18].

\section{REFERENCES}

[1] F. Akyildiz, W. Su, Y. Sankarasubramaniam, E. Cayirici, A survey on sensor networks, IEEE communications magazine, 40 (8) (2002) 102-114.

[2] M. Zareei, M.I. Alghamdi, A.K.M.M. Islam, S. Baharun, R. Budiarto, "Energy Efficient Handover Management in Cluster Based Wireless Sensor Network" in Proceeding of the Electrical Engineering Computer Science and Informatics (2014). 
[3] F. Liang, L. Zhang, P. Sun, "Study on the Rough-set-based Clustering Algorithm for Sensor Networks", in Bulletin of Electrical Engineering and Informatics, (2014).

[4] V. Mhatre, C. Rosenberg, Design guidelines for wireless sensor networks: communication, clustering and aggregation, Ad Hoc Network Journal, 2 (1) (2004) 45-63.

[5] G.J. Pottie and W.J. Kaiser, "Wireless Integrated Network Sensors", Communications of the ACM, vol. 43, no. 5, May 2000, pp. 51-58.

[6] Y.C. Tseng, S.Y. Ni, Y.S. Chen, and J.P. Sgeu, "The broadcast storm problem in a mobile ad hoc network", The Journal of Mobile Communication Computation and Information, vol. 8, no. 2-3, pp. 153-167, 2002. View at Publisher - View at Google Scholar J.

[7] $\mathrm{Li}$ and $\mathrm{P}$. Mohapatra, "An analytical model for the energy hole problem in many-to-one sensor networks", in Proceedings of the 62nd IEEE Vehicular Technology Conference, vol. 4, pp. 2721-2725, 2005. View at Publisher View at Google Scholar.

[8] W.R. Heinzelman, A.P. Chandrakasan, and H. Bala-Krishnan, "An Application-specific Protocol Architecture for Wireless Microsensor Networks", IEEE Transactions on Wireless Communications, 1, No. 4, pp. 660-670, 2002.

[9] E. Shih, S. Cho, N. Ickes, R. Min, A. Sinha, A. Wang, A. Chandrakasan, "Physical Layer Driven Protocol and Algorithm Design for Energy-Efficient Wireless Sensor Networks", ACM SIGMOBILE 7/01 Rome, Italy.

[10] J. Hill, R. Szewczyk, A. Woo, S. Hollar, D. Culler, K. Pister. "Architecture Directions for Networked Sensors", ASPLOSIX 11/00 Cambridge, MA, USA.

[11] J. Polastre, J. Hill, D. Culler, "Versatile Low Power Media Access for Wireless Sensor Networks", SenSys'04, Baltimore, Maryland, USA, November 3-5, 2004.

[12] V. Shnayder, M. Hempstead, B. Chen, G. Werner Allen, and M. Welsh, "Simulating the Power Consumption of LargeScale Sensor Network Applications", SenSys'04, Baltimore, Maryland, USA, November 3-5, 2004.

[13] Mille, M.J. and N.H. Vaidya, "A mac protocol to reduce sensor network energy consumption using a wakeup radio", IEEE Trans. Mobile Computing, Vol. 4, No. 3, 228-242, May 2005.

[14] M.N. Halgamuge, M. Zukerman, and K. Ramamohanarao "AN ESTIMATION OF SENSOR ENERGY CONSUMPTION", Progress In Electromagnetics Research B, Vol. 12, 259-295, 2009.

[15] M. Abo-Zahhad, M. Farrag, A. Ali and O. Amin, "An energy consumption model for wireless sensor networks, " Energy Aware Computing Systems \& Applications (ICEAC), 2015 International Conference on, Cairo, 2015, pp.14.

[16] "MICA2 mote datasheet", https://www.eol.ucar.edu/rtf/facilities/isa/internal/CossBow/DataSheets/mic2.pdf.

2004,

[online]

[17] http://www.xbow.com/ Products/Product-pdf files/Wireless-pdf/MICA2-Datasheet.pdf.

[18] Zhu, J. and S. Papavassiliou, "On the energy-efficient organization and the lifetime of multi-hop sensor networks", IEEE Commun. Lett., Vol. 7, No. 11, 537-539, Nov. 2003. 